\title{
ALL MEAGER FILTERS MAY BE NULL
}

\author{
TOMEK BARTOSZYNSKI, MARTIN GOLDSTERN, \\ HAIM JUDAH, AND SAHARON SHELAH
}

(Communicated by Andreas R. Blass)

\begin{abstract}
We show that in the Cohen model the sum of two nonmeasurable sets is always nonmeager. As a consequence we show that it is consistent with ZFC that all filters which have the Baire property are Lebesgue measurable. We also show that the existence of a Sierpinski set implies that there exists a nonmeasurable filter which has the Baire property.
\end{abstract}

\section{INTRODUCTION}

The goal of this paper is to show yet another example of nonduality between measure and category.

Suppose that $\mathscr{F}$ is a nonprincipal filter on $\omega$. Identify $\mathscr{F}$ with the set of characteristic functions of its elements. Under this convention $\mathscr{F}$ becomes a subset of $2^{\omega}$ and a question about its topological or measure-theoretical properties makes sense.

It has been proved by Sierpinski that every nonprincipal filter has either Lebesgue measure zero or is nonmeasurable. Similarly it is either meager or does not have the Baire property.

In [T] Talagrand proved that

Theorem 1.1. There exists a measurable filter that does not have the Baire property.

In fact we have an even stronger result. In $[\mathrm{Ba}]$ it is proved that

Theorem 1.2. Every measurable filter can be extended to a measurable filter that does not have the Baire property.

We show that the dual result is false.

Received by the editors February 18, 1991 and, in revised form, June 12, 1991.

1991 Mathematics Subject Classification. Primary 03E35; Secondary 04A20.

The first author thanks the Lady Davis Fellowship Trust for full support.

The fourth author's research was partially supported by Basic Research Fund, Israel Academy of Sciences, Publication 434. 


\section{A MODEL WHERE ALL MEAGER FILTERS ARE NULL}

In this section we prove the following theorem.

Theorem 2.1. It is consistent with ZFC that every filter that has the Baire property is measurable.

Proof. We will use the following more general result.

Theorem 2.2. Let $\mathbf{V} \models G C H$ and suppose that $\mathbf{V}[G]$ is a generic extension of $\mathbf{V}$ obtained adding $\omega_{2}$ Cohen reals. Then in $\mathbf{V}[G]$ for any two sets $A, B \subseteq 2^{\omega}$ if $A+B=\{a+b: a \in A, b \in B\}$ is a meager set then either $A$ or $B$ has measure zero.

Proof. Note that we apply this lemma only for the case $A=B$. Therefore to simplify the notation we assume that $A=B$. The proof of the general case is almost the same. We follow [Bu].

We will use the following notation. Let

$$
F n(X, 2)=\left\{s: \operatorname{dom}(s) \in[X]^{<\omega} \text { and } \operatorname{range}(s) \subseteq\{0,1\}\right\}
$$

be the notion of forcing adding $|X|$-many Cohen reals. For $s \in F n(X, 2)$ let $[s]=\left\{f \in 2^{X}: s \subseteq f\right\}$.

Let $\mathbf{V} \models G C H$ be a model of ZFC and $G_{\omega_{2}}$ be an $F n\left(\omega_{2}, 2\right)$-generic filter over $\mathbf{V}$. Clearly $c=\bigcup G_{\omega_{2}}$ is a generic sequence of $\omega_{2}$ Cohen reals and $\mathbf{V}[c]=\mathbf{V}\left[G_{\omega_{2}}\right]$.

Let $\left\{F_{n}: n \in \omega\right\}$ be a sequence of closed, nowhere dense sets such that $A+A \subseteq \bigcup_{n \in \omega} F_{n}$. Without loss of generality we can assume that $\left\{F_{n}: n \in \omega\right\}$ $\in \mathbf{V}$.

Let $\left\{a_{\xi}: \xi<\omega_{2}\right\}$ be an enumeration of all elements of $A$. For every $\xi<\omega_{2}$ let $\dot{a}_{\xi}$ be a name for $a_{\xi}$. In other words for every $\xi<\omega_{2}$ we have a countable set $I_{\xi} \subseteq \omega_{2}$ such that $\dot{a}_{\xi}$ is a Borel function from $2^{I_{\xi}}$ into $2^{\omega}$. Moreover $a_{\xi}$ is the value of the function $\dot{a}_{\xi}$ on Cohen reals, i.e., $\dot{a}_{\xi}\left(c \mid I_{\xi}\right)=a_{\xi}$. In addition we can find a dense $G_{\delta}$ set $H_{\xi} \subseteq 2^{I_{\xi}}$ such that $\dot{a}_{\xi} \mid H_{\xi}$ is a continuous function.

For $\alpha, \xi, \eta<\omega_{2}$ define $\xi \simeq_{\alpha} \eta$ if

(1) $I_{\xi}$ and $I_{\eta}$ are order isomorphic,

(2) the order-isomorphism between $I_{\xi}$ and $I_{\eta}$ transfers $\dot{a}_{\xi}$ onto $\dot{a}_{\eta}$ and $H_{\xi}$ onto $H_{\eta}$,

(3) $I_{\xi} \cap \alpha=I_{\eta} \cap \alpha$.

Notice that for every $\alpha<\omega_{2}$ the relation $\simeq_{\alpha}$ is an equivalence relation with $\omega_{1}$ many equivalence classes.

Lemma 2.3. There exists $\alpha^{\star}<\omega_{2}$ such that

$$
\forall \xi, \beta \exists \eta\left(\xi \simeq_{\alpha^{\star}} \eta \& I_{\eta} \cap\left(\beta-\alpha^{\star}\right)=\varnothing\right) .
$$

Proof. Let $M$ be an elementary substructure of a sufficiently large part of the universe (say, $M \prec H\left(\aleph_{434}\right)$ ) of size $\aleph_{1}$ containing all relevant information (i.e., $\omega_{1} \subseteq M,\left\langle\dot{a}_{\xi}: \xi<\omega_{2}\right\rangle \in M$, etc.).

Let $\alpha^{\star}=M \cap \omega_{2}$. $\alpha^{\star}$ is an ordinal of cofinality $\omega_{1}$. We will show that $\alpha^{\star}$ satisfies the conclusion of the lemma.

Take any $\xi<\omega_{2}$ and any $\beta$. If $\beta<\alpha^{\star}$ or $I_{\xi} \subseteq \alpha^{\star}$, then we can choose $\eta=\xi$. So assume $\beta \geq \alpha^{\star}$ and $I_{\xi}-\alpha^{\star} \neq \varnothing$. There is $\alpha_{0}<\alpha^{\star}$ such that 
$I_{\xi} \cap \alpha_{0}=I_{\xi} \cap \alpha^{\star}$. Let $E_{0}=[\xi]_{\alpha_{0}}$ be the $\simeq_{\alpha_{0}}$-equivalence class of $\xi$ and $\mathscr{E}$ be the set of all $\simeq_{\alpha_{0}}$-equivalence classes.

Since $\mathscr{E} \in M$, also the set

$$
S=\left\{\sup \left\{\min \left(I_{\eta}-\alpha_{0}\right): \eta \in E\right\}: E \in \mathscr{E}\right\}
$$

is in $M$. As $|S| \leq \aleph_{1}, S \subseteq M$.

Since $\min \left(I_{\xi}-\alpha_{0}\right)>\alpha^{\star}$, we also have $\sup \left\{\min \left(I_{\eta}-\alpha_{0}\right): \eta \in E_{0}\right\}>\alpha^{*}$. As $S \cap\left[\alpha^{\star}, \omega_{2}\right)=\varnothing$, we conclude

$$
\sup \left\{\min \left(I_{\eta}-\alpha_{0}\right): \eta \in E_{0}\right\}=\omega_{2},
$$

hence there is $\eta \in E_{0}$ with $\min \left(I_{\eta}-\alpha_{0}\right) \geq \beta$, i.e., $I_{\eta} \cap\left(\beta-\alpha_{0}\right)=\varnothing$.

So $I_{\xi} \cap \alpha^{\star}=I_{\xi} \cap \alpha_{0}=I_{\eta} \cap \alpha_{0}=I_{\eta} \cap \alpha^{\star}$, where the last equality holds because $I_{\eta} \cap\left(\alpha^{\star}-\alpha_{0}\right) \subseteq I_{\eta} \cap\left(\beta-\alpha_{0}\right)=\varnothing$. Also $I_{\eta} \cap\left(\beta-\alpha^{\star}\right) \subseteq I_{\eta} \cap\left(\beta-\alpha_{0}\right)=\varnothing$.

Let $\alpha^{\star}$ be the ordinal from the above lemma. Work in $\mathbf{V}^{\prime}=\mathbf{V}\left[c \mid \alpha^{\star}\right]$. For every $\xi<\omega_{2}$ define

$$
D_{\xi}=\left\{s \in F n\left(\omega_{2}-\alpha^{\star}, 2\right): \operatorname{cl}\left(\dot{a}_{\xi}([s])\right) \text { has measure zero }\right\} .
$$

Lemma 2.4. $D_{\xi}$ is dense in $F n\left(\omega_{2}-\alpha^{\star}, 2\right)$ for every $\xi<\omega_{2}$.

Proof. Notice that it is enough to show that $D_{\xi} \cap F n\left(I_{\xi}-\alpha^{\star}, 2\right)$ is dense in $F n\left(I_{\xi}-\alpha^{\star}, 2\right)$ for $\xi<\omega_{2}$.

Suppose that this fails. Find $\xi<\omega_{2}$ and $s_{0} \in F n\left(I_{\xi}-\alpha^{\star}, 2\right)$ such that for all $s \supseteq s_{0}$ the set $\operatorname{cl}\left(\dot{a}_{\xi}([s])\right)$ has positive measure.

Using the lemma with $\beta>\sup \left(I_{\xi}\right)$ we can find $\eta<\omega_{2}$ such that $\xi \simeq_{\alpha^{\star}} \eta$ and $\left(I_{\xi}-\alpha^{\star}\right) \cap\left(I_{\eta}-\alpha^{\star}\right)=\varnothing$. Notice that there exists $t_{0} \in F n\left(I_{\eta}-\alpha^{\star}, 2\right)$ (the image of $s_{0}$ under the isomorphism between $I_{\xi}$ and $I_{\eta}$ ) such that for every $t \supseteq t_{0}$ the set $\operatorname{cl}\left(\dot{a}_{\eta}([t])\right)$ has positive measure.

Since $s_{0}$ and $t_{0}$ have disjoint domains, $s_{0} \cup t_{0} \in F n\left(\omega_{2}-\alpha^{\star}, 2\right)$. Find $n \in \omega$ and a condition $u \in F n\left(\omega_{2}-\alpha^{\star}, 2\right)$ extending $s_{0} \cup t_{0}$ such that $u \Vdash$ $\dot{a}_{\xi}(\dot{c})+\dot{a}_{\eta}(\dot{c}) \in F_{n} . u$ can then be written as $u_{1} \cup u_{2} \cup u_{3}$ where $s_{0} \subseteq u_{1} \in$ $F n\left(I_{\xi}-\alpha^{\star}, 2\right), t_{0} \subseteq u_{2} \in F n\left(I_{\eta}-\alpha^{\star}, 2\right)$, and $u_{3} \in F n\left(\omega_{2}-\left(I_{\xi} \cup I_{\eta} \cup \alpha^{\star}\right), 2\right)$. By the assumption the sets $\operatorname{cl}\left(\dot{a}_{\xi}\left(\left[u_{1}\right]\right)\right), \operatorname{cl}\left(\dot{a}_{\eta}\left(\left[u_{2}\right]\right)\right)$ have positive measure. By a well-known theorem of Steinhaus the set $\operatorname{cl}\left(\dot{a}_{\xi}\left(\left[u_{1}\right]\right)\right)+\operatorname{cl}\left(\dot{a}_{\eta}\left(\left[u_{2}\right]\right)\right)$ contains an open set (hence also $\left(\operatorname{cl}\left(\dot{a}_{\xi}\left(\left[u_{1}\right]\right)\right)+\operatorname{cl}\left(\dot{a}_{\eta}\left(\left[u_{2}\right]\right)\right)\right)-F_{n}$ contains an open set). Using the fact that $\dot{a}_{\xi}$ and $\dot{a}_{\eta}$ are continuous functions we can find $u_{1} \subseteq$ $s_{1} \in F n\left(I_{\xi}-\alpha^{\star}, 2\right)$ and $u_{2} \subseteq t_{1} \in F n\left(I_{\eta}-\alpha^{\star}, 2\right)$ such that $\left(\operatorname{cl}\left(\dot{a}_{\xi}\left(\left[s_{1}\right]\right)\right)+\right.$ $\left.\operatorname{cl}\left(\dot{a}_{\eta}\left(\left[t_{1}\right]\right)\right)\right) \cap F_{n}=\varnothing$. But this is a contradiction since

$$
s_{1} \cup t_{1} \cup u_{3} \Vdash \dot{a}_{\xi}(\dot{c})+\dot{a}_{\eta}(\dot{c}) \in F_{n} \text {. }
$$

Notice that for $\xi<\omega_{2}$

$$
\begin{aligned}
& D_{\xi}=\left\{s \in F n\left(I_{\xi}\right):\right. \text { there exists a closed measure zero } \\
& \left.\qquad \text { set } F \in V \text { such thats } \Vdash-\dot{a}_{\xi}(\dot{c}) \in F\right\} .
\end{aligned}
$$

Therefore by the above lemma

$$
A \subseteq \bigcup\left\{F: F \text { is a closed measure zero set coded in } \mathbf{V}^{\prime}\right\} .
$$


Since $\mathbf{V}$ contains Cohen reals over $\mathbf{V}^{\prime}$, the union of all closed measure zero sets coded in $\mathbf{V}^{\prime}$ has measure zero in $\mathbf{V}$. We conclude that $A$ has measure zero.

Let $\mathscr{F}$ be a nonprincipal filter. Denote by $\mathscr{F} c=\{X \subseteq \omega: \omega-X \in \mathscr{F}\}$. $\mathscr{F}^{c}$ is an ideal and it is very easy to see that $\mathscr{F}$ is measurable (has the Baire property) iff $\mathscr{F}^{c}$ is measurable (has the Baire property).

Lemma 2.5. $\mathscr{F}+\mathscr{F}=\mathscr{F}^{c}$.

Proof. Suppose that $X, Y \in \mathscr{F}$. Then

$$
\{n: X(n)+Y(n)=0\} \supseteq X^{-1}(1) \cap Y^{-1}(1) \in \mathscr{F} .
$$

In general $\mathscr{F}+\cdots+\mathscr{F}$ is equal to $\mathscr{F}$ or $\mathscr{F} c$ depending on whether there is an even or odd number of $\mathscr{F}$ 's.

Let $\mathbf{V} \vDash G C H$ and suppose that $\mathbf{V}[G]$ is a generic extension of $\mathbf{V}$ obtained by adding $\omega_{2}$ Cohen reals. By the above lemma if $\mathscr{F}$ is a meager filter then $\mathscr{F}^{c}=\mathscr{F}+\mathscr{F}$ is meager. So by $2.2 \mathscr{F}$ has measure zero.

\section{Filters that ARE MEAGER AND NONMEASURABle}

Theorem 2.1 shows that in order to construct a filter that is meager and nonmeasurable we need some extra assumptions.

In [T] Talagrand showed that

Theorem 3.1. Suppose that the real line is not the union of $<2^{\aleph_{0}}$ many measure zero sets. Then there exists a nonmeasurable filter which is meager.

Let $\kappa$ be a regular uncountable cardinal. Recall that $S$ is a generalized Sierpinski set of size $\kappa$ if $|S \cap H|<\kappa$ for every null set $H$. It is clear that all $S^{\prime} \subseteq S$ of size $\kappa$ are also nonmeasurable. Note that the assumptions of the above theorem imply the existence of a generalized Sierpinski set of size $2^{\aleph_{0}}$.

Theorem 3.2. Assume that there exists a generalized Sierpinski set. Then there exists a nonmeasurable meager filter.

Proof. Let $S$ be a generalized Sierpinski set of size $\kappa$. Build a sequence $\left\{x_{\xi}\right.$ : $\xi<\kappa\} \subseteq S$ and an elementary chain of models $\left\{M_{\xi}: \xi<\kappa\right\}$ of size $\kappa$ such that

(1) $\left\{x_{\xi}: \xi<\alpha\right\} \subseteq M_{\alpha}$ for $\alpha<\kappa$,

(2) $x_{\beta}$ is a random real over $M_{\alpha}$ for $\beta>\alpha$.

Suppose that $M_{\beta}, x_{\beta}$ are already constructed for $\beta<\alpha$. Since $S$ is a Sierpinski set,

$$
\bigcup\left\{S \cap H: H \text { is a null set coded in } M_{\beta} \text { for } \beta<\alpha\right\}
$$

has size $<\kappa$. Let $x_{\alpha}$ be any element of $S$ avoiding this set.

Let $X_{\xi}=x_{\xi}^{-1}(1)$ for $\xi<\kappa$. Let $\mathscr{F}$ be the filter generated by the family $\left\{X_{\xi}: \xi<\kappa\right\}$. We will show that $\mathscr{F}$ has the required properties. 
For $X \subseteq \omega$ let

$$
d(X)=\lim _{n \rightarrow \infty}|X \cap n| / n
$$

if the above limit exists.

By easy induction we show that we have $d\left(X_{\xi_{1}} \cap \cdots \cap X_{\xi_{n}}\right)=2^{-n}$ for $\xi_{1}, \ldots$, $\xi_{n}<\kappa$. This shows that

$$
\mathscr{F} \subseteq\left\{X \subseteq \omega: \liminf _{n \rightarrow \infty} \frac{|X \cap n|}{n}>0\right\},
$$

which is a meager set. To check that $\mathscr{F}$ is nonmeasurable notice that $\mathscr{F}$ contains the nonmeasurable set $\left\{x_{\xi}: \xi<\kappa\right\}$.

It is an open problem whether one can construct a meager nonmeasurable filter assuming the existence of a nonmeasurable set of size $\aleph_{1}$. We only have some partial results.

Let $\mathfrak{b}$ be the size of the smallest unbounded family in $\omega^{\omega}$, and let unif be the size of the smallest nonmeasurable set.

For $X \subseteq \omega$ let $f_{X} \in \omega^{\omega}$ be an increasing function enumerating $X$. For a filter $\mathscr{F}$ let $\mathscr{F}^{\star}=\left\{f_{X}: X \in \mathscr{F}\right\}$. In $[\mathrm{J}]$ it is proved that

Theorem 3.3. For every filter $\mathscr{F}$,

\section{$\mathscr{F}$ has the Baire property iff $\mathscr{F}^{\star}$ is bounded.}

Theorem 3.4. Suppose that unif $<\mathfrak{b}$. Then there exists a nonmeasurable filter that is meager.

Proof. Let $X \subseteq 2^{\omega}$ be a nonmeasurable set of size unif. Let $M$ be a model of the same size containing $X$ as a subset. Then $M \cap 2^{\omega}$ does not have measure zero, so it is nonmeasurable. Consider any filter $\mathscr{F}$ such that $M \models$ $\mathscr{F}$ is an ultrafilter. $\mathscr{F}$ generates a filter in $\mathbf{V}$ and this filter is meager by 3.3 and the fact that it is generated by unif $<\mathfrak{b}$ many elements. On the other hand $M \models 2^{\omega}=\mathscr{F} \cup \mathscr{F}^{c}$ and we know that $M \cap 2^{\omega}$ is a nonmeasurable set. Hence $\mathscr{F}$ is nonmeasurable.

The previous theorem depended on the implication:

If $\mathscr{F}$ has measure zero then $M \cap 2^{\omega}$ has measure zero.

This implication is not true in general for any set $X \in M$ having outer measure 1 in $M$ as is showed by the following example.

Example. It is consistent with ZFC that there are models $M \subseteq \mathbf{V}$ such that only some sets which have outer measure 1 in $M$ have measure 0 in $V$.

Let $\mathbf{V}=\mathbf{L}[c]\left[\left\langle r_{\xi}: \xi<\omega_{1}\right\rangle\right]$ where $c$ is a Cohen real over $\mathbf{L}$ and $\left\langle r_{\xi}: \xi<\right.$ $\left.\omega_{1}\right\rangle$ is a sequence of random reals over $\mathbf{L}[c]$ (added side by side). Let $M=$ $\mathbf{L}\left[\left\langle r_{\xi}: \xi<\omega_{1}\right\rangle\right]$. Consider the set $X=\mathbf{L} \cap 2^{\omega}$. It is known that $X$ is a nonmeasurable set in $M$ but $X$ has measure 0 in $\mathbf{V}$. On the other hand the set $\left\{r_{\xi}: \xi<\omega_{1}\right\}$ is nonmeasurable in $\mathbf{V}$. 
We conclude the paper with an example of a filter that is destroyed by any new real. This was proved independently by Woodin.

Recall that some ultrafilters are preserved (generate ultrafilters) in forcing extensions. In particular there is a large class of proper partial orders that preserve $p$-points. It contains Sacks forcing, rational perfect set forcing and is closed under countable support iteration (see [BS]).

The next theorem shows that no forcing notion (adding new reals) preserves all ultrafilters.

Theorem 3.5. Let $M$ be a model for ZFC and let $r$ be a real that does not belong to $M$. Then there exists a filter $\mathscr{F}$ such that $M \models \mathscr{F}$ is an ultrafilter but

$$
M[r] \vDash\{X \subseteq \omega: \exists Y \in \mathscr{F} Y \subseteq X\} \text { is not an ultrafilter. }
$$

Proof. Let $\left\{k_{n}: n \in \omega\right\}$ be a fast increasing sequence of natural numbers. Let $T$ be a tree on $2^{<\omega}$ such that:

(1) $\forall s \in T \exists n\left(|s|=k_{n}\right) \leftrightarrow s \frown 0 \in T \& s \frown 1 \in T$.

(2) Let $\left\{s_{1}, \ldots, s_{2^{n}}\right\}$ be the list of $T \cap 2^{k_{n}}$ in lexicographical order. Then for every $w \subseteq \mathscr{P}\left(2^{n}\right)-\left\{\varnothing, 2^{n}\right\}$ there exists $m \in\left(k_{n-1}, k_{n}\right)$ such that $s_{l}(m)=0$ iff $l \in w$.

(3) There is no $m \in \omega$ such that for all $s \in T \cap 2^{m+1}$ we have $s(m)=0$ or for all $s \in T \cap 2^{m+1}$ we have $s(m)=1$.

Let $S \subseteq T$ be a subtree of $T$. Define

$$
\begin{aligned}
& A_{S}^{0}=\left\{m: \forall s \in S \cap 2^{m+1} s(m)=0\right\}, \\
& A_{S}^{1}=\left\{m: \forall s \in S \cap 2^{m+1} s(m)=1\right\} .
\end{aligned}
$$

Let $\mathcal{J}$ be the ideal generated by sets $\left\{A_{S}^{0}, A_{S}^{1}: S\right.$ is a perfect subtree of $\left.T\right\}$.

One can easily verify that all finite subsets of $\omega$ belong to $\mathscr{J}$.

Lemma 3.6. $\mathcal{J}$ is a proper ideal.

Proof. Let $S_{1}, \ldots, S_{m}$ be perfect subtrees of $T$. Find $n$ sufficiently big so that $\left|S_{j} \cap 2^{k_{n}}\right|>m$ for $j \leq m$. Let $s_{1}, \ldots, s_{2^{n}}$ be the list of $T \cap 2^{k_{n}}$ in lexicographical ordering. Let $w_{1}, \ldots, w_{m}$ be such that $S_{j} \cap 2^{k_{n}}=\left\{s_{i}: i \in w_{j}\right\}$ for $j \leq m$. Let $w=\left\{\min \left(w_{1}\right), \ldots, \min \left(w_{m}\right)\right\}$. Then for all $j, w_{j} \nsubseteq w$ and $w_{j} \cap w \neq \varnothing$. By the definition of $T$ there is $k_{n-1}<k<k_{n}$ such that $w=\left\{l: s_{l}(k)=0\right\}$. By the property of $w$ for every $j \leq m$ there exist $s^{0}, s^{1} \in S_{j} \cap 2^{k_{n}}$ such that $s^{0}(k)=0$ and $s^{1}(k)=1$. Therefore $k \notin A_{S_{1}}^{0} \cup$ $A_{S_{1}}^{1} \cup \cdots \cup A_{S_{m}}^{0} \cup A_{S_{m}}^{1}$.

Let $\mathscr{F}$ be any ultrafilter in $M$ extending the filter $\{\omega-X: X \in \mathscr{J}\}$. Let $r$ be a real that does not belong to $M$. Without loss of generality we can assume that $r$ is a branch through $T$.

Assume that $\mathscr{F}$ generates an ultrafilter, and let $X_{r}=\{n: r(n)=1\}$. We can assume that there exists an element $X \in \mathscr{F}$ such that $X \subseteq X_{r}$. Let $S=\{s \in T: \forall k \in X(|s|>k \rightarrow s(k)=1)\}$. Clearly $r$ is a branch through $S$. But in that case $S$ contains a perfect subtree $S_{1} \subseteq S$ (since it contains a new branch). Therefore $X \subseteq A_{S_{1}}^{1} \in \mathcal{J}$, a contradiction. 


\section{REFERENCES}

[Ba] T. Bartoszynski, On the structure of the filters on a countable set, Real Anal. Exchange 17 (1992).

[BS] A. Blass and S. Shelah, There may be simple $P_{\aleph_{1}}$ - and $P_{\aleph_{2}}$-points and the Rudin-Keisler ordering may be downward directed, Ann. Pure Appl. Logic 33 (1987).

[Bu] M. Burke, notes of June 17, 1989.

[J] H. Judah, Unbounded filters on $\omega$, Logic Colloquium, North-Holland, Amsterdam and New York, 1987.

[T] M. Talagrand, Compacts de fonctions mesurables et filtres nonmesurables, Studia Math. 67 (1980).

(T. Bartoszynski) Department of Mathematics, Boise State University, Boise, Idaho 83725

E-mail address: tomek@math.idbsu.edu

(M. Goldstern and H. Judah) Department of Mathematics, Bar Ilan University, Ramat GAN, 52900 ISRAEL

E-mail address, M. Goldstern: goldstrn@bimacs.cs.biu.ac.il

E-mail address, H. Judah: judah@bimacs.cs.biu.ac.il

(S. Shelah and T. Bartosznski) Department of Mathematics, Hebrew University, JerUSALEM, 91904 ISRAEL

E-mail address, S. Shelah: shelah@sunrise.huji.ac.il 